Pacific

Journal of

Mathematics

MULTIPLICITY OF SOLUTIONS TO THE YAMABE PROBLEM ON COLLAPSING RIEMANNIAN SUBMERSIONS

Renato G. Bettiol and Paolo Piccione 


\title{
MULTIPLICITY OF SOLUTIONS TO THE YAMABE PROBLEM ON COLLAPSING RIEMANNIAN SUBMERSIONS
}

\author{
Renato G. Bettiol and Paolo Piccione
}

\begin{abstract}
Let $g_{t}$ be a family of constant scalar curvature metrics on the total space of a Riemannian submersion obtained by shrinking the fibers of an original metric $g$, so that the submersion collapses as $t \rightarrow 0$ (that is, the total space converges to the base in the Gromov-Hausdorff sense). We prove that, under certain conditions, there are at least 3 unit volume constant scalar curvature metrics in the conformal class $\left[g_{t}\right]$ for infinitely many $t$ accumulating at 0 . This holds, for instance, for homogeneous metrics $g_{t}$ obtained via Cheeger deformation of homogeneous fibrations with fibers of positive scalar curvature.
\end{abstract}

\section{Introduction}

A classic problem in Riemannian geometry is to find possible canonical metrics on a given smooth manifold $M$. Along this quest, an important achievement was the complete solution of the celebrated Yamabe problem, which states that given a closed Riemannian manifold $\left(M, g_{0}\right)$ with $\operatorname{dim} M \geq 3$, there exists a constant scalar curvature metric $g$ conformal to $g_{0}$. Such a metric $g$ can be characterized variationally as a critical point of the Hilbert-Einstein functional

$$
\mathscr{A}(g)=\frac{1}{\operatorname{Vol}(g)} \int_{M} \operatorname{scal}(g) \operatorname{vol}_{g}
$$

restricted to the set $\left[g_{0}\right]_{1}$ of unit volume metrics in the conformal class of $g_{0}$. Existence of a metric that minimizes this constrained functional, called the Yamabe metric, is a consequence of the works of Yamabe [1960], Trudinger [1968], Aubin [1976] and Schoen [1984]. In addition to the minimizer, there may also be other critical points; thus the solution may be not unique. However, Anderson [2005]

The second named author is supported by FAPESP, São Paulo, Brazil, and by CNPq, Brazil. This paper was concluded during Bettiol's visit to the University of São Paulo in May 2012. We thankfully acknowledge the financial support for this visit from FAPESP, processo 2011/21362-2, "Group actions, submanifold theory and global analysis in Riemannian and pseudo-Riemannian geometry". MSC2010: primary 53C30, 58J55; secondary 53A30, 53C20, 53C21, 58E50, 58J50.

Keywords: Yamabe problem, constant scalar curvature metrics, equivariant bifurcation, Riemannian submersions, Cheeger deformation, homogeneous fibration. 
recently proved that, on generic conformal classes, the Yamabe metric is the unique solution. In the present paper, we are interested in the complementary situation, that is, finding conformal classes where the Yamabe problem has multiple solutions. Our main results provide a large class of manifolds whose conformal class contains at least 3 distinct solutions (see Theorems A and B).

A classic method to obtain new solutions of a PDE from a path of known solutions is to use bifurcation theory. The basic setup for our framework consists of a given one-parameter family $g_{t}$ of known solutions to the Yamabe problem,

$$
\mathrm{d}\left(\left.\mathscr{A}\right|_{\left[g_{t}\right]_{1}}\right)\left(\hat{g}_{t}\right)=0 \quad \text { for } t \in[a, b],
$$

where $\hat{g}_{t}$ is the unit volume metric homothetic to $g_{t}$. We study the case where $g_{t}$ is obtained by shrinking the fibers of a Riemannian submersion with totally geodesic fibers. By proving that certain topological invariants (for example, the Morse index) of $g_{t}$ change as $t$ crosses a value $t_{*}$, one obtains existence of new solutions accumulating at $g_{t_{*}}$. Then, a simple trick (Proposition 7.1) implies that for $t$ 's close to $t_{*}$ there are at least 3 solutions to the Yamabe problem on $\left[g_{t}\right]_{1}$.

In our main result, $g_{t}$ are homogeneous metrics, hence trivially solutions to the Yamabe problem and good (that is, nongeneric) candidates for admitting other solutions in their conformal class. Let $H \subsetneq K \subsetneq G$ be compact connected Lie groups with $\operatorname{dim} K / H \geq 2$, and assume that either $H$ is normal in $K$ or $K$ is normal in $G$. Consider the homogeneous fibration

$$
K / H \rightarrow G / H \stackrel{\pi}{\rightarrow} G / K, \quad \pi(g H)=g K
$$

More precisely, $\pi$ is the associated bundle with fiber $K / H$ to the $K$-principal bundle $G \rightarrow G / H$. Endow the above spaces with compatible homogeneous metrics (see Section 4). Shrinking the fibers of (1-3) by a factor $t^{2}$, we get a family $g_{t}$ of homogeneous metrics on $G / H$, sometimes called canonical variation of (1-3). This is, up to reparametrization, the Cheeger deformation of $G / H$ in the direction of the natural $K$-action. As $t$ approaches 0 , the manifolds $\left(G / H, g_{t}\right)$ converge to the base $G / K$ in the Gromov-Hausdorff sense. We explore the existence of infinitely many bifurcations of (1-2) along this collapse of $\left(G / H, g_{t}\right)$ onto the base to obtain the following multiplicity result.

Theorem A. Let $K / H \rightarrow G / H \rightarrow G / K$ be a homogeneous fibration endowed with compatible homogeneous metrics such that $K / H$ has positive scalar curvature. Let $g_{t}$ be the family of $G$-invariant metrics on $G / H$ obtained as described above. Then, there exists a subset $\mathcal{T} \subset] 0,1[$, accumulating at 0 , such that for each $t \in \mathcal{T}$ there are at least 2 solutions to the Yamabe problem in $\left[g_{t}\right]_{1}$, other than $\hat{g}_{t}$, and they are not $G$-invariant. 
Theorem A applies, for example, to short exact sequences $K \rightarrow G \rightarrow G / K$ of compact connected Lie groups and to twisted product fibrations

$$
K / H \rightarrow((K \times L) / \Gamma) / H \rightarrow G / K,
$$

where $K / H$ is a compact homogenous space of positive scalar curvature and $G=(K \times L) / \Gamma$ is a connected compact Lie group. More interestingly, the result also holds, for example, for any homogeneous fibrations (1-3) where $H$ is normal in $K$ and the quotient $K / H$ is a nonabelian compact connected Lie group. In this case, $G$ can have arbitrarily large rank and dimension, and the corresponding possible total spaces can be much more general than twisted products. For more details and examples, see Section 5. We also stress that the hypotheses made on the scalar curvature (and dimension) of the fibers $K / H$ are necessary. A counterexample is given in the end of Section 4.

Various other nonuniqueness phenomena have been studied in the literature, but usually this can only be achieved for very specific examples; see [Ambrosetti and Malchiodi 1999; Berti and Malchiodi 2001; Bettiol and Piccione 2013; Fischer and Marsden 1975; Lima et al. 2012; Schoen 1991]. One exception is a remarkable result of Pollack [1993] that proved existence of arbitrarily $C^{0}$-small perturbations of any given metric, with an arbitrarily large number of solutions in its conformal class. Previously, Schoen [1991] had proven the existence of an increasing number of solutions, with larger energy and Morse index, in the conformal class of the product $S^{1}(r) \times S^{m-1}$ of round spheres, as $r$ tends to infinity. Lima, Piccione and Zedda [Lima et al. 2012] generalized this result to families of product metrics on a product $M_{1} \times M_{2}$ of compact Riemannian manifolds given by rescaling one of the factors, obtaining bifurcation of solutions. Inspired by these results, the authors recently obtained similar bifurcation results for families of homogeneous spheres in [Bettiol and Piccione 2013]. The core of Theorem A is a further generalization, establishing that such bifurcations indeed occur on several other families of compact homogeneous spaces.

The initial approach to detect bifurcation in a variational problem, such as (1-2), is to look for a change in the Morse index. This is done by identifying eigenvalues of the second variation (2-3) of $\left.\mathscr{A}\right|_{\left[g_{t}\right]_{1}}$ that change sign for certain values of $t$. Nevertheless, a subtle compensation problem may occur when other eigenvalues with the same combined multiplicity cross zero in the opposite direction. On the one hand, it is not hard to detect passage through zero of certain explicitly computable parts of the spectrum. On the other hand, it is in general not possible to rule out that other eigenvalues also change sign at the same time, potentially producing this compensation that leaves the Morse index unchanged.

In the case of the Yamabe problem, the spectral analysis required consists of comparing eigenvalues of the Laplacian of Riemannian submersions with the 
scalar curvature. Since the fibers are assumed totally geodesic, eigenfunctions of the Laplacian of the base may be lifted to eigenfunctions of the total space that are constant along the fibers (see Section 3). This provides an easier subset of the spectrum to deal with, in the sense that a direct computation of the scalar curvature immediately gives infinitely many crossings through zero. Nevertheless, no information is available in general regarding possible compensation due to other crossings in the opposite direction.

The key to handle this situation is to use homogeneity, placing the problem in an equivariant context where a subtler bifurcation criterion due to Smoller and Wasserman [1990] applies. Linearizing the action, one gets a representation of the symmetry group in each eigenspace of the second variation. The direct sum of those representations that correspond to negative eigenvalues is called the negative isotropic representation; see page 8 . The equivariant criterion asserts that bifurcation occurs at the degeneracy values where the negative isotropic representation changes, which is the case of all degeneracy values mentioned above that correspond to crossings of eigenvalues of the base. It is then a simple observation that such bifurcation yields the desired multiplicity result (see Proposition 7.1).

Although homogeneity is strongly used in our main result, one can replace it by other hypotheses that make it possible to avoid the compensation problem. The general context is then a Riemannian submersion with totally geodesic fibers,

$$
F \rightarrow M \stackrel{\pi}{\rightarrow} B
$$

and metrics $g_{t}$ on $M$, obtained by shrinking the fibers. Unfortunately, in general, these alternative hypotheses are quite restrictive. Since avoiding this compensation is the central issue in our results, it is natural to expect that a deeper understanding of this issue would allow weaker hypotheses; see Remark 6.2. One possibility is to impose curvature conditions that imply certain lower bounds on eigenvalues of the Laplacian, in which case bifurcation is obtained via the easier Morse index criterion.

Theorem B. Let $F \rightarrow M \rightarrow B$ be a Riemannian submersion with totally geodesic fibers. Let $l=\operatorname{dim} F \geq 2$ and $m=\operatorname{dim} M$. Assume the metrics $g_{t}$ obtained by shrinking the fibers have constant scalar curvature 1 and that for some $\tau>0$ and $k_{1}, k_{2}>0$,

$$
\left\{\begin{array} { l } 
{ \operatorname { R i c } _ { F } \geq ( l - 1 ) k _ { 1 } } \\
{ \operatorname { s c a l } _ { F } < l ( m - 1 ) k _ { 1 } }
\end{array} \quad \text { and } \left\{\begin{array}{l}
\operatorname{Ric}_{\left(M, g_{\tau}\right)} \geq(m-1) k_{2} \\
\operatorname{scal}_{B} \leq m(m-1) k_{2} .
\end{array}\right.\right.
$$

There exists an infinite subset $\mathcal{T}$ of positive real numbers accumulating at 0 , such that for each $t \in \mathcal{T}$ there are at least 3 solutions to the Yamabe problem in the conformal class $\left[g_{t}\right]$.

\footnotetext{
${ }^{1}$ For example, this happens if the original metric on $M$ is Einstein; see [Besse 1987, Corollary 9.62].
} 
The paper is organized as follows. In Section 2, we briefly review the variational setup for the Yamabe problem and the bifurcation techniques (Propositions 2.2 and 2.3) introduced in [Bettiol and Piccione 2013; Lima et al. 2012]. The effect of shrinking the fibers on the spectrum of a Riemannian submersion with totally geodesic fibers is recalled in Section 3. The core of the proof of Theorem A (Theorem 4.1) is given in Section 4. Section 5 describes several examples to which these theorems apply. Section 6 contains the core of the proof of Theorem B (Theorem 6.1). Finally, Section 7 explains how to translate bifurcation of solutions into the multiplicity results claimed above.

\section{Variational framework and bifurcation criteria}

We start by briefly recalling the classic variational setup for the Yamabe problem; see [Bettiol and Piccione 2013; Lima et al. 2012; Schoen 1989] for details. Let $M$ be a closed smooth manifold of dimension $m$. Consider the set $\operatorname{Met}(M)$ of $C^{r, \alpha}$ Riemannian metrics on $M$, which is an open convex cone in the Banach space of $C^{r, \alpha}$ symmetric $(0,2)$-tensors. Henceforth we fix $r \geq 3$ and $\left.\alpha \in\right] 0,1[$. For each $g \in \operatorname{Met}(M)$, define its $C^{r, \alpha}$ conformal class by

$$
[g]=\left\{\phi g: \phi \in C^{r, \alpha}(M), \phi>0\right\} .
$$

Denote by $\operatorname{Met}_{1}(M)$ the smooth codimension 1 embedded submanifold of $\operatorname{Met}(M)$ formed by unit volume metrics. Finally, let

$$
[g]_{1}=\operatorname{Met}_{1}(M) \cap[g] .
$$

The set $[g]_{1}$ is a codimension 1 Banach submanifold of $[g]$, and its tangent space at the metric $g$ can be canonically identified as

$$
T_{g}[g]_{1} \cong\left\{\psi \in C^{r, \alpha}(M): \int_{M} \psi \operatorname{vol}_{g}=0\right\} .
$$

The choice of Hölder regularity $C^{r, \alpha}$ for the metric tensors and functions is due to a technical analytic aspect of our theory that employs certain Schauder estimates. To simplify the exposition, these technicalities will not be discussed in further detail.

Hilbert-Einstein functional. Fix a metric $g_{0}$ on the manifold $M$ and consider the Hilbert-Einstein functional $\mathscr{A}$ defined in (1-1). The restriction of $\mathscr{A}$ to $\left[g_{0}\right]_{1}$ is smooth, and its critical points are the constant scalar curvature metrics in $\left[g_{0}\right]_{1}$. Given one such critical point $g \in\left[g_{0}\right]_{1}$, the second variation of $\mathscr{A}$ at $g$ can be identified with the quadratic form on (2-1) given by

$$
\mathrm{d}^{2}\left(\left.\mathscr{A}\right|_{[g]_{1}}\right)(g)(\psi, \psi)=\frac{m-2}{2} \int_{M}\left((m-1) \Delta_{g} \psi-\operatorname{scal}(g) \psi\right) \psi \operatorname{vol}_{g},
$$


where $\Delta_{g}$ is the Laplace-Beltrami operator of $g$, or Laplacian of $g$, with the sign convention such that its spectrum is nonnegative. The above quadratic form is represented by the (formally) self-adjoint elliptic operator

$$
J_{g}(\psi)=\frac{(m-1)(m-2)}{2}\left(\Delta_{g} \psi-\frac{\operatorname{scal}(g)}{m-1} \psi\right),
$$

which we call the Jacobi operator at $g$. From the above formulas, the critical point $g \in\left[g_{0}\right]_{1}$ is nondegenerate (in the usual sense of Morse theory) if and only if $\operatorname{scal}(g) /(m-1)$ is not an eigenvalue of the Laplacian $\Delta_{g}$, or if $\operatorname{scal}(g)=0$. Moreover, the Morse index $N(\mathrm{~g})$ of this critical point is given by the number of negative eigenvalues of (2-3), that is, the number of positive eigenvalues of $\Delta_{g}$, counted with multiplicity, that are less than $\operatorname{scal}(g) /(m-1)$. More precisely, denote by

$$
0<\lambda_{1}^{g} \leq \lambda_{2}^{g} \leq \lambda_{3}^{g} \leq \cdots \leq \lambda_{j}^{g} \leq \cdots
$$

the sequence of eigenvalues of the Laplacian $\Delta_{g}$, repeated according to their multiplicity. Then, the Morse index of $g$ is given by

$$
N(g)=\max \left\{j \in \mathbb{N}: \lambda_{j}^{g}<\frac{\operatorname{scal}(g)}{m-1}\right\} .
$$

Remark 2.1. For the purposes of this paper, the relevant data are the signs of the eigenvalues of the operator (2-3). Note that, given $\alpha>0$, one has $\Delta_{\alpha g}=\frac{1}{\alpha} \Delta_{g}$ and $\operatorname{scal}(\alpha g)=\frac{1}{\alpha} \operatorname{scal}(g)$. Hence, the spectrum of (2-3) scales in a trivial way under homotheties, in the sense that negative (respectively positive) eigenvalues remain negative (respectively positive). On the other hand, $\operatorname{vol}_{\alpha g}=\alpha^{m / 2} \operatorname{vol}_{g}$. Thus, whenever necessary, we may renormalize a metric to have unit volume without compromising the above spectral theory.

A classic result of variational bifurcation theory. Let us turn to the main tool used in this paper, bifurcation theory. Consider a continuous path $g_{t} \in \operatorname{Met}(M)$ of solutions to the Yamabe problem, as in (1-2). A value $t_{*} \in[a, b]$ is a bifurcation value for the family $g_{t}$ if there exists a sequence $\left\{t_{q}\right\}$ in $[a, b]$ that converges to $t_{*}$ and a sequence $\left\{g_{q}\right\}$ in $\operatorname{Met}(M)$ that converges to $g_{t_{*}}$ satisfying for all $q \in \mathbb{N}$ :

(i) $g_{t_{q}} \in\left[g_{q}\right]$, but $g_{q} \neq g_{t_{q}}$.

(ii) $\operatorname{Vol}\left(g_{q}\right)=\operatorname{Vol}\left(g_{t_{q}}\right)$.

(iii) $\operatorname{scal}\left(g_{q}\right)$ is constant.

If $t_{*} \in[a, b]$ is not a bifurcation value, then the family $g_{t}$ is locally rigid at $t_{*}$. In other words, the family $g_{t}$ is locally rigid at $t_{*} \in[a, b]$ if there exists a neighborhood $U$ of $g_{t_{*}}$ in $\operatorname{Met}(M)$ such that, for $t \in[a, b]$ sufficiently close to $t_{*}$, the conformal 
class $\left[g_{t}\right]$ contains a unique metric of constant scalar curvature in $U$ whose volume equals the volume of $g_{t}$.

Using a suitable version of the Implicit Function Theorem, one sees that if $g_{t_{*}}$ is a nondegenerate critical point, then $g_{t}$ is locally rigid at $t_{*}$; see [Lima et al. 2012, Proposition 3.1]. Thus, degeneracy is a necessary condition for bifurcation, however it is in general not sufficient. A classic result in variational bifurcation theory states that, given a continuous path of smooth functionals and a continuous path of critical points, there is a bifurcating branch issuing from the given path at each point where the Morse index changes (see [Kielhöfer 2004, Theorem II.7.3] or [Smoller and Wasserman 1990, Theorem 2.1]). Translating this result to our variational framework, we get the following.

Proposition 2.2 [Lima et al. 2012, Theorem 3.3]. Let $[a, b] \ni t \mapsto g_{t} \in \operatorname{Met}(M)$ be a continuous path of constant scalar curvature metrics on $M$, and assume that $a$ and $b$ are not degeneracy values for $g_{t}$. If $N\left(g_{a}\right) \neq N\left(g_{b}\right)$, then there exists a bifurcation value $\left.t_{*} \in\right] a, b\left[\right.$ for the family $g_{t}$.

Equivariant variational bifurcation. In many applications, the criterion of Proposition 2.2 cannot be employed because establishing a change of the Morse index at a given degeneracy value may be a difficult task. However, when the variational setup has an equivariant nature, one can replace the change of Morse index condition with a more general condition based on the representation theory of the group of symmetries of the variational problem. This more general condition fits perfectly the setup discussed in the present paper, and we now describe it in the variational framework of the Yamabe problem.

Suppose there exists a finite-dimensional nice ${ }^{2}$ Lie group $K$ that acts (on the left) by diffeomorphisms on a compact manifold $M$, and let $[a, b] \ni t \mapsto g_{t} \in \operatorname{Met}(M)$ be a continuous path of constant scalar curvature metrics on $M$. Up to a suitable normalization, let us assume that each $g_{t}$ has unit volume (see Remark 2.1). The $K$-action on $M$ induces a (right) $K$-action on $\operatorname{Met}(M)$ by pull-back; that is, the action of $k \in K$ on $g \in \operatorname{Met}(M)$ is $k^{*} g$. Assume that the $K$-action on $M$ is isometric with respect to all metrics $g_{t}$, that is,

\footnotetext{
${ }^{2}$ A group $G$ is nice in the sense of [Smoller and Wasserman 1990] if, given unitary representations $\pi_{1}$ and $\pi_{2}$ of $G$ on Hilbert spaces $V_{1}$ and $V_{2}$ respectively, such that $B_{1}\left(V_{1}\right) / S_{1}\left(V_{1}\right)$ and $B_{1}\left(V_{2}\right) / S_{1}\left(V_{2}\right)$ have the same (equivariant) homotopy type as $G$-spaces, then $\pi_{1}$ and $\pi_{2}$ are unitarily equivalent. Here, $B_{1}$ and $S_{1}$ denote respectively the unit ball and the unit sphere in the specified Hilbert space, and the quotient $B_{1}\left(V_{i}\right) / S_{1}\left(V_{i}\right)$ is meant in the topological sense (that is, it denotes the unit ball of $V_{i}$ with its boundary contracted to one point).

For example, any compact connected Lie group $G$ is nice. More generally, any compact Lie group with less than 5 connected components is nice. Denoting by $G^{0}$ the identity connected component of $G$, then $G$ is nice if the discrete part $G / G^{0}$ is either the product of a finite number of copies of $\mathbb{Z}_{2}$ (for example, the case $G=\mathrm{O}(n)$ ); or the product of a finite number of copies of $\mathbb{Z}_{3}$; or if $G / G^{0}=\mathbb{Z}_{4}$.
} 


$$
k^{*}\left(g_{t}\right)=g_{t} \quad \text { for all } k \in K, t \in[a, b] .
$$

In this situation, the $K$-action on $\operatorname{Met}(M)$ leaves every conformal class [ $\left.g_{t}\right]$ invariant, and also $\left[g_{t}\right]_{1}$ is $K$-invariant for all $t$. Note that (2-5) means that, for all $t, g_{t}$ is a fixed point of the $K$-action on $\operatorname{Met}(M)$.

It is easy to see that, given $\phi: M \rightarrow \mathbb{R}$ a positive function,

$$
\mathscr{A}\left(\phi^{4 /(n-2)} g_{t}\right)=\int_{M}\left(4 \frac{n-1}{n-2} \phi \Delta_{t} \phi+\operatorname{scal}\left(g_{t}\right) \phi^{2}\right) \operatorname{vol}_{g_{t}},
$$

where $\Delta_{t}$ is the Laplacian of $g_{t}$. Using that $k^{*}\left(\phi g_{t}\right)=(\phi \circ k) g_{t}$, right-composition with isometries commutes with $\Delta_{t}$ and $\operatorname{scal}\left(g_{t}\right)$ is constant, it follows from a change of variables that

$$
\mathscr{A}\left(k^{*}\left(\phi g_{t}\right)\right)=\mathscr{A}\left(\phi g_{t}\right) \quad \text { for all } k \in K, t \in[a, b] .
$$

Thus, denoting by $\mathscr{A}_{t}$ the restriction of the Hilbert-Einstein functional (1-1) to the conformal class $\left[g_{t}\right]_{1}$, we have that $\mathscr{A}_{t}$ is invariant under the $K$-action on $\left[g_{t}\right]_{1}$.

For each eigenvalue $\lambda \in \operatorname{Spec}\left(\Delta_{t}\right)$, denote by $E_{t}^{\lambda} \subset L^{2}\left(M\right.$, $\left.\operatorname{vol}_{g_{t}}\right)$ the correspondent eigenspace. Elements of $E_{t}^{\lambda}$ are smooth functions, and $\operatorname{dim} E_{t}^{\lambda}<+\infty$ is the multiplicity of $\lambda$. It is easy to see that, for every $t \in[a, b]$ and $\lambda \in \operatorname{Spec}\left(\Delta_{t}\right)$, there is a representation $\pi_{t}^{\lambda}$ of $K$ in $E_{t}^{\lambda}$, given by right-composition with isometries:

$$
\pi_{t}^{\lambda}(k) \psi=\psi \circ k \quad \text { for all } k \in K, \psi \in E_{t}^{\lambda} .
$$

Note this is (the restriction to $E_{t}^{\lambda}$ of) the isotropy representation of the $K$-action on $\left[g_{t}\right]_{1}$ at the fixed point $g_{t}$, that is, the linearization of this $K$-action at $g_{t}$. We remark that since the $K$-action by pull-back on $\left[g_{t}\right]_{1}$ is a right action, its linearization at a fixed point is actually an antirepresentation. However, we will henceforth not make distinctions between left/right actions and representations/antirepresentations since this does not affect our arguments.

Denote by $\mathcal{N}_{t}$ the set of negative eigenvalues of the operator (2-3); that is,

$$
\left.\mathcal{N}_{t}:=\operatorname{Spec}\left(J_{g_{t}}\right) \cap\right]-\infty, 0[.
$$

Now, for all $t \in[a, b]$, define the negative isotropic representation $\pi_{t}^{-}$of $K$ in $\bigoplus_{t} E_{t}^{\lambda}$ as the direct sum representation $\lambda \in \mathcal{N}_{t}$

$$
\pi_{t}^{-}:=\bigoplus_{\lambda \in \mathcal{N}_{t}} \pi_{t}^{\lambda}
$$

Notice that the degree ${ }^{3}$ of $\pi_{t}^{-}$is always finite and equal to the Morse index $N\left(g_{t}\right)$ defined in (2-4). Finally, recall that two representations $\pi_{i}: K \rightarrow \operatorname{GL}\left(V_{i}\right), i=1,2$,

\footnotetext{
${ }^{3}$ That is, the dimension of the vector space $\bigoplus_{\lambda \in \mathcal{N}_{t}} E_{t}^{\lambda}$.
} 
are isomorphic if there exists a $K$-equivariant isomorphism $T: V_{1} \rightarrow V_{2}$, that is, such that $\pi_{2}(k) \circ T=T \circ \pi_{1}(k)$ for all $k \in K$.

Proposition 2.3. Let $K$ be a nice Lie group acting on a compact manifold $M$ and $[a, b] \ni t \mapsto g_{t} \in \operatorname{Met}(M)$ be a continuous path of (unit volume) constant scalar curvature metrics. Suppose the $K$-action is isometric on $\left(M, g_{t}\right)$ for all $t \in[a, b]$. If $a$ and $b$ are not degeneracy values for $g_{t}$, and if the negative isotropic representations $\pi_{a}^{-}$and $\pi_{b}^{-}$are not isomorphic, then there exists a bifurcation value $\left.t_{*} \in\right] a, b\left[\right.$ for the family $g_{t}$.

Proof. This result is a direct application of an equivariant bifurcation result due to Smoller and Wasserman [1990, Theorem 3.1]. More precisely, one needs a slightly more general statement of the result, applied to functionals defined on a varying manifold. In our case, the functional is the Hilbert-Einstein functional $\mathscr{A}$, defined on the varying manifold $\left[g_{t}\right]_{1}$. Details can be found in [Lima et al. 2012, Theorem 3.4, Theorem A.2].

Notice the above is a refinement of Proposition 2.2, which corresponds to saying that $\pi_{a}^{-}$and $\pi_{b}^{-}$do not have the same degree (and hence cannot be isomorphic).

\section{Laplacian on collapsing Riemannian submersions}

In order to study bifurcation from an initial family $g_{t}$ of solutions to the Yamabe problem on $M$, it is crucial to have a good understanding of the spectra of their Laplacians $\Delta_{t}$. In all our applications, the family $g_{t}$ is obtained as a deformation of the original metric $g$ on the total space $M$ of a Riemannian submersion with totally geodesic fibers (1-4), by multiplying it by a factor $t^{2}$ in the direction of the fibers. We will be particularly interested in the behavior of the spectrum of $\Delta_{t}$ as the fibers collapse to a point, that is, as $t \rightarrow 0$.

The effect of such deformation on the spectrum was first studied by BérardBergery and Bourguignon [1982], where $g_{t}$ is called canonical variation of $g$. The starting point is that (1-4) remains a Riemannian submersion with totally geodesic fibers when $g$ is replaced by $g_{t}$; see [Bérard-Bergery and Bourguignon 1982, Proposition 5.2]. For the readers' convenience, we briefly recall some related results that are discussed in more detail in [Bettiol and Piccione 2013, Section 3].

Vertical Laplacian and lifts of eigenfunctions. Define the vertical Laplacian $\Delta_{v}$ on a function $\psi: M \rightarrow \mathbb{R}$ by

$$
\left(\Delta_{v} \psi\right)(p):=\left(\left.\Delta_{F} \psi\right|_{F_{p}}\right)(p) \text { for all } p \in M,
$$

where $\Delta_{F}$ is the Laplacian of the fiber and $F_{p}=\pi^{-1}(\pi(p))$ is the fiber through $p \in M$. Just like a usual Laplacian, the vertical Laplacian is a nonnegative selfadjoint unbounded operator on $L^{2}(M)$, however it is not elliptic (unless $\pi$ is a 
covering). Since the fibers are isometric, $\Delta_{v}$ has a discrete spectrum equal to that of the Laplacian $\Delta_{F}$ of the fiber. Let us denote

$$
\begin{aligned}
\operatorname{Spec}\left(\Delta_{M}\right) & =\left\{0=\mu_{0}<\mu_{1}<\cdots<\mu_{k} \nearrow+\infty\right\}, \\
\operatorname{Spec}\left(\Delta_{v}\right) & =\left\{0=\phi_{0}<\phi_{1}<\cdots<\phi_{j} \nearrow+\infty\right\},
\end{aligned}
$$

where these eigenvalues are not repeated according to their multiplicity. Note that the multiplicity of the eigenvalues of $\Delta_{M}$ is always finite, but the eigenvalues of $\Delta_{v}$ might have infinite multiplicity. For instance, $\Delta_{v} \widetilde{\psi}=0$ implies only that $\widetilde{\psi}$ is constant along the fibers; that is, $\widetilde{\psi}=\psi \circ \pi$ for some function $\psi: B \rightarrow \mathbb{R}$ on the base.

It is easy to see that, for any $\psi: B \rightarrow \mathbb{R}$ and its lift $\widetilde{\psi}:=\psi \circ \pi$,

$$
\Delta_{M} \widetilde{\psi}=\left(\Delta_{B} \psi\right) \circ \pi+g\left(\operatorname{grad}_{g} \widetilde{\psi}, \vec{H}\right),
$$

where $\vec{H}$ is the mean curvature vector field of the fibers. Since we assumed the fibers of $\pi$ are totally geodesic, $\vec{H}$ vanishes identically. Thus, if $\psi$ is an eigenfunction of $\Delta_{B}$, then its lift $\widetilde{\psi}$ is an eigenfunction of $\Delta_{M}$ with the same eigenvalue (and constant along the fibers). Therefore, there is a natural inclusion

$$
\operatorname{Spec}\left(\Delta_{B}\right) \subset \operatorname{Spec}\left(\Delta_{M}\right)
$$

Conversely, if $\psi: M \rightarrow \mathbb{R}$ is constant along the fibers and satisfies $\Delta_{M} \psi=\lambda \psi$, then there exists $\breve{\psi}: B \rightarrow \mathbb{R}$ such that $\Delta_{B} \breve{\psi}=\lambda \breve{\psi}$ and $\psi=\breve{\psi} \circ \pi$. Summing up, it follows from (3-2), after checking the adequate regularity hypotheses (see [Bessa et al. 2012, Lemma 3.11]), that the following holds.

Proposition 3.1. If $\pi: M \rightarrow B$ is a Riemannian submersion with totally geodesic fibers, then an eigenfunction of $\Delta_{M}$ is constant along the fibers if and only if it is the lift of an eigenfunction of $\Delta_{B}$.

Spectrum of deformed metrics. Another consequence of having totally geodesic fibers is that $L^{2}(M)$ has a Hilbert basis consisting of simultaneous eigenfunctions of the original Laplacian $\Delta_{M}$ and the vertical Laplacian $\Delta_{v}$; see [Bérard-Bergery and Bourguignon 1982, Theorem 3.6]. This means that these operators can be simultaneously diagonalized, in the appropriate sense. Furthermore, it is a simple calculation to show that that the Laplacian $\Delta_{t}$ of the deformed metric $g_{t}$ is $\Delta_{t}=$ $\Delta_{M}+\left(1 / t^{2}-1\right) \Delta_{v}$; see [Bérard-Bergery and Bourguignon 1982, Proposition 5.3]. From this, we get the following description of its spectrum.

Proposition 3.2 [Bettiol and Piccione 2013, Corollary 3.6]. For each $t>0$, the following inclusion holds:

$$
\operatorname{Spec}\left(\Delta_{t}\right) \subset \operatorname{Spec}\left(\Delta_{M}\right)+\left(1 / t^{2}-1\right) \operatorname{Spec}\left(\Delta_{v}\right) .
$$


Since these sets are discrete, every eigenvalue $\lambda(t)$ of $\Delta_{t}$ is of the form

$$
\lambda^{k, j}(t)=\mu_{k}+\left(1 / t^{2}-1\right) \phi_{j},
$$

for some eigenvalues $\mu_{k}$ and $\phi_{j}$ of $\Delta_{M}$ and $\Delta_{v}$, respectively.

Remark 3.3. Not all possible combinations of $\mu_{k}$ and $\phi_{j}$ in (3-4) give rise to an eigenvalue of $\Delta_{t}$. In fact, this only happens when the total space of the submersion is a Riemannian product. In general, determining which combinations are allowed depends on the global geometry of the submersion.

Note that, since the fibers of $\pi$ remain totally geodesic with respect to $g_{t},(3-3)$ holds when $\Delta_{M}$ is replaced with $\Delta_{t}$; that is,

$$
\operatorname{Spec}\left(\Delta_{B}\right) \subset \operatorname{Spec}\left(\Delta_{t}\right) \text { for all } t>0 .
$$

Moreover, when $j=0$ in (3-4), $\lambda^{k, 0}(t)=\mu_{k} \in \operatorname{Spec}\left(\Delta_{M}\right)$ remains an eigenvalue of $\Delta_{t}$ for $t \neq 1$ if and only if $\mu_{k} \in \operatorname{Spec}\left(\Delta_{B}\right)$. Such eigenvalues $\lambda^{k, 0}(t)$ of $\Delta_{t}$ will be called constant eigenvalues, since they are independent of $t$. In other words, the constant eigenvalues of $\Delta_{t}$ are the ones in the left-hand side of (3-5). We stress that $\lambda^{k, 0}(t)$ is not necessarily a constant eigenvalue for all $k$.

\section{Bifurcation on homogeneous fibrations}

Let $H \subsetneq K \subsetneq G$ be compact connected Lie groups such that $\operatorname{dim} K / H \geq 2$, and assume that either $H$ is normal in $K$, or $K$ is normal in $G$. Consider the homogeneous fibration (1-3),

$$
K / H \rightarrow G / H \stackrel{\pi}{\rightarrow} G / K, \quad \text { where } \pi(g H)=g K,
$$

and notice that the fiber over $g K \in G / K$ is $(g K) H \subset G / H$. Define a $K$-action on $G / H$ by $k \cdot g H=k g H$ if $K$ is normal in $G$ and by $k \cdot g H=g k^{-1} H$ if $H$ is normal in $K$. Notice that the orbits of this $K$-action are exactly the fibers of $\pi$. Denote by $\mathfrak{h} \subsetneq \mathfrak{k} \subsetneq \mathfrak{g}$ the Lie algebras of $H \subsetneq K \subsetneq G$. We henceforth fix an $\operatorname{Ad}_{G}(K)$-invariant complement $\mathfrak{m}$ to $\mathfrak{k}$ in $\mathfrak{g}$, and an $\operatorname{Ad}_{G}(H)$-invariant complement $\mathfrak{p}$ to $\mathfrak{h}$ in $\mathfrak{k}$; that is,

$$
\mathfrak{k} \oplus \mathfrak{m}=\mathfrak{g}, \quad[\mathfrak{k}, \mathfrak{m}] \subset \mathfrak{m} \quad \text { and } \quad \mathfrak{h} \oplus \mathfrak{p}=\mathfrak{k}, \quad[\mathfrak{h}, \mathfrak{p}] \subset \mathfrak{p} .
$$

There are natural identifications of $\mathfrak{m}$ and $\mathfrak{p}$ with the tangent spaces to $G / K$ and $K / H$ at the origin, ${ }^{4}$ respectively. The sum $\mathfrak{m} \oplus \mathfrak{p}$ is an $\operatorname{Ad}_{G}(H)$-invariant complement to $\mathfrak{h}$ in $\mathfrak{g}$, which is identified with the tangent space to $G / H$ at the origin.

Any $\operatorname{Ad}_{G}(K)$-invariant inner product on $\mathfrak{m}$ defines a $G$-invariant metric on the base $G / K$, and any $\operatorname{Ad}_{G}(H)$-invariant inner product on $\mathfrak{p}$ defines a $K$-invariant

\footnotetext{
${ }^{4}$ That is, $\mathfrak{m} \cong T_{(e K)} G / K$ and $\mathfrak{p} \cong T_{(e H)} K / H$.
} 
metric on the fiber $K / H$. The orthogonal direct sum of these inner products on $\mathfrak{m} \oplus \mathfrak{p}$ now gives a $G$-invariant metric on $G / H$. We call these metrics on (1-3) compatible homogeneous metrics. We stress that not necessarily all $G$-invariant metrics on $G / H$ arise in this way. However, if $\mathfrak{m}$ and $\mathfrak{p}$ do not share equivalent $\operatorname{Ad}_{G}(H)$ submodules, then all $G$-invariant metrics on $G / H$ that project to a $G$-invariant metric on $G / K$ are of this form. We will henceforth consider all homogeneous fibrations to be endowed with compatible metrics. The homogeneous fibration $\pi: G / H \rightarrow G / K$ is then automatically a Riemannian submersion with totally geodesic fibers (isometric to $K / H$ ); see [Bérard-Bergery 1978, Proposition 2] or [Besse 1987, Theorem 9.80].

Theorem 4.1. Let $K / H \rightarrow G / H \rightarrow G / K$ be a homogeneous fibration as above, and assume that $K / H$ has positive scalar curvature. Let $g_{t}$ be the family of homogeneous metrics on $G / H$ obtained by scaling the fibers by $t^{2}$. There exists a sequence $\left\{t_{q}\right\}$ in $] 0,1\left[\right.$, that converges to 0 , of bifurcation values for the family $g_{t}$.

Proof. Since $\pi:\left(G / H, g_{t}\right) \rightarrow G / K$ is a Riemannian submersion with totally geodesic fibers, we have

$$
\operatorname{scal}\left(G / H, g_{t}\right)=\frac{1}{t^{2}} \operatorname{scal}(K / H)+\operatorname{scal}(G / K) \circ \pi-t^{2}\|A\|^{2},
$$

where $\|A\|$ is the Hilbert-Schmidt norm of the Gray-O'Neill tensor of integrability of the horizontal distribution (see [Besse 1987, Proposition 9.70]). Note that $\operatorname{scal}(K / H)>0$ implies

$$
\lim _{t \rightarrow 0_{+}} \operatorname{scal}\left(G / H, g_{t}\right)=+\infty
$$

Recall that the degeneracy values in this setup are those $t>0$ such that

$$
(m-1) \Delta_{t} \psi-\operatorname{scal}\left(G / H, g_{t}\right) \psi=0
$$

has a nontrivial solution $\psi$, where $m=\operatorname{dim} G / H$ and $\Delta_{t}$ is the Laplacian of $\left(G / H, g_{t}\right)$. From (3-4) and (4-1), the set of such degeneracy values is discrete. From (4-2), there are infinitely many degeneracy values $t_{q}$ accumulating at 0 such that $\operatorname{scal}\left(G / H, g_{t_{q}}\right) /(m-1) \in \operatorname{Spec}\left(\Delta_{G / K}\right) \subset \operatorname{Spec}\left(\Delta_{t}\right)$; see (3-5). We claim that every such value $t_{q}$ is a bifurcation value.

Fix one such $t_{q}$, and denote by $\lambda \in \operatorname{Spec}\left(\Delta_{G / K}\right)$ the constant eigenvalue of $\Delta_{t}$ such that $\operatorname{scal}\left(G / H, g_{t_{q}}\right) /(m-1)=\lambda$. If there is a change in the Morse index at $t_{q}$, that is, for $\varepsilon>0$ sufficiently small, $N\left(g_{t_{q}-\varepsilon}\right) \neq N\left(g_{t_{q}+\varepsilon}\right)$, then by Proposition $2.2, t_{q}$ is a bifurcation value. However, if the Morse index does not change, there must be a compensation of eigenvalues. Namely, there must exist nonconstant eigenvalues $\lambda^{k_{1}, j_{1}}(t), \ldots, \lambda^{k_{n}, j_{n}}(t)$ of $\Delta_{t}$, whose combined multiplicity equals the multiplicity 
of $\lambda$, such that
$\lambda<\operatorname{scal}\left(G / H, g_{t}\right) /(m-1)<\lambda^{k_{i}, j_{i}}(t)$ for all $t<t_{q}$ (close to $\left.t_{q}\right)$ and $1 \leq i \leq n$,
$\lambda>\operatorname{scal}\left(G / H, g_{t}\right) /(m-1)>\lambda^{k_{i}, j_{i}}(t)$ for all $t>t_{q}$ (close to $\left.t_{q}\right)$ and $1 \leq i \leq n$.

Denoting by $E_{t}^{\alpha}$ the eigenspace of the eigenvalue $\alpha \in \operatorname{Spec}\left(\Delta_{t}\right)$, we have the negative isotropic representations $\pi_{t}^{-}$on the linear spaces (of same finite dimension):

$$
\begin{array}{ll}
E \oplus E_{t}^{\lambda} & \text { for } t<t_{q}\left(\text { close to } t_{q}\right), \\
E \oplus \bigoplus_{i} E_{t}^{\lambda^{k_{i}, j_{i}}} & \text { for } t>t_{q}\left(\text { close to } t_{q}\right),
\end{array}
$$

where $E$ is the space spanned by the eigenfunctions with eigenvalues less than $\operatorname{scal}\left(G / H, g_{t}\right) /(m-1)$ for $t$ close to $t_{q}$. We claim that for small $\varepsilon>0$, the negative isotropic representations $\pi_{t_{q}-\varepsilon}^{-}$and $\pi_{t_{q}+\varepsilon}^{-}$on the spaces (4-4) cannot be isomorphic. From Proposition 2.3, it then follows that $t_{q}$ is a bifurcation value, concluding the proof.

Let us verify the above claim. Given a representation $\pi$ of a compact group, denote by $\mathfrak{I}(\pi)$ the number of copies of the trivial representation in the irreducible decomposition of $\pi$. It is easily seen that a necessary condition for the two representations $\pi_{a}$ and $\pi_{b}$ to be isomorphic is that $\mathfrak{I}\left(\pi_{a}\right)=\mathfrak{I}\left(\pi_{b}\right)$. For the negative isotropic representation $\pi_{t}^{-}$, one can compute

$$
\mathfrak{I}\left(\pi_{t}^{-}\right)=\sum_{\substack{\eta \in \operatorname{Spec}\left(\Delta_{G / K}\right) \\ \eta<\operatorname{scal}\left(g_{t}\right) /(m-1)}} \operatorname{mul}(\eta),
$$

where $\operatorname{mul}(\eta)$ is the multiplicity of $\eta$ as an eigenvalue of $\Delta_{G / K}$. Indeed, an eigenfunction $\psi$ of $\Delta_{t}$ is constant along the fibers $K / H$ of the homogeneous fibration (1-3) if and only if it is $K$-invariant, that is, $\psi \circ k=\psi$ for all $k \in K$. This is equivalent to saying that $\psi$ is a fixed point of $\pi_{t}^{-}$; see (2-6). So, from Proposition 3.1, the left-hand side of (4-5) is greater than or equal to the right-hand side. Conversely, it is easy to see that if $\psi: G / H \rightarrow \mathbb{R}$ is the linear combination of eigenfunctions $\psi_{i}: G / H \rightarrow \mathbb{R}$ of $\Delta_{t}$, and if $\psi$ is constant along the fibers of $G / H \rightarrow G / K$, then each $\psi_{i}$ must be constant along such fibers. This follows from the fact that the subspace of $L^{2}(G / H)$ of functions that are constant along the fibers (which is isomorphic to $L^{2}(G / K)$ ) is spanned by the (lift of) eigenfunctions of $\Delta_{G / K}$. In other words, the space spanned by the eigenfunctions of $\Delta_{G / H}$ that are constant along the fibers and the space spanned by the eigenfunctions of $\Delta_{G / H}$ that are not constant along the fibers are $L^{2}$-orthogonal. This completes the proof of (4-5).

From (4-4) and (4-5) we see that, for any $\varepsilon>0$ small, $\Im\left(\pi_{t_{q}-\varepsilon}^{-}\right)>\Im\left(\pi_{t_{q}+\varepsilon}^{-}\right)$. Therefore these representations are not isomorphic, concluding the proof. 
Remark 4.2. At all bifurcation values for the family $g_{t}$ a break of symmetry occurs, in the sense that any solutions in the bifurcating branch are not $G$-homogeneous. This follows easily from the fact that each conformal class contains at most one homogeneous metric (up to rescaling).

Sharpness of fiber hypotheses. If the fibers $K / H$ have flat scalar curvature or have dimension 1 , then $\operatorname{scal}\left(g_{t}\right)$ remains bounded as $t \rightarrow 0$, and there are not infinitely many degeneracy values as above. For instance, consider a fibration of tori, $G=K \times K, K=T^{2}$ and $H=\{e\}$, where the inclusion of $K$ is as one of the factors of $G$. If $K$ is endowed with the flat metric and $G$ with the product metric, shrinking the fibers keeps the total space $G / H$ flat, hence the family obtained is (trivially) locally rigid, for all $t>0$.

\section{Examples}

We now discuss how to construct examples of homogeneous fibrations with fibers of positive scalar curvature to which Theorem 4.1 (hence also Theorem A) applies.

Normal homogeneous metrics. A $K$-invariant metric on $K / H$ is called normal if it is obtained from the restriction to $\mathfrak{p}$ of a bi-invariant inner product on $\mathfrak{k}$. Since $K$ is compact, it admits a bi-invariant metric. Hence, normal homogeneous metrics always exist ${ }^{5}$ on $K / H$. Endowed with such a metric, the sectional curvature of a tangent plane at the origin, spanned by orthonormal vectors $X$ and $Y$, is

$$
\sec (X, Y)=\frac{1}{4}\|[\bar{X}, \bar{Y}]\|^{2}+\frac{3}{4}\left\|[\bar{X}, \bar{Y}]_{\mathfrak{h}}\right\|^{2} \geq 0,
$$

where $\bar{X}=(0, X)$ and $\bar{Y}=(0, Y)$ are the horizontal lifts of $X$ and $Y$ to $\mathfrak{k}=\mathfrak{h} \oplus \mathfrak{p}$ and $(\cdot)_{\mathfrak{h}}$ denotes the $\mathfrak{h}$-component of a vector in $\mathfrak{k}$. In particular, $\operatorname{scal}(K / H) \geq 0$, and it is equal to zero if and only if $\mathfrak{p}$ is an abelian ideal of $\mathfrak{k}$. This, in turn, is equivalent to the existence of an abelian subgroup $A \subset K$ that acts transitively on $K / H$. Since (the closure of) $A$ is a compact connected abelian Lie group, it must be a torus. Hence, $K / H$ itself is a torus. Thus, any normal metric on $K / H$ has positive scalar curvature, unless $K / H$ is a torus.

More generally, $K / H$ admits a (normal) metric of positive scalar curvature if and only if its universal covering is not diffeomorphic to an Euclidean space; see [Bérard-Bergery 1978, Theorem 2]. In particular, if $K / H$ is not an aspherical manifold, then $K / H$ has a metric of positive scalar curvature. We also stress that, generally, there are other $K$-invariant metrics (not necessarily normal) on $K / H$ that have positive scalar curvature, and any such metric can be used to obtain

\footnotetext{
${ }^{5}$ There exist homogeneous spaces on which all homogeneous metrics are normal. These spaces are called of normal type in [Bérard-Bergery 1978, Section 7]. Examples are spaces whose isotropy representation is irreducible (for example, irreducible symmetric spaces) and their products.
} 
examples of applications of our results. In this direction, examples with nonnormal homogeneous metrics with positive scalar curvature on spheres will be discussed below.

In any of the cases above, one can endow the remaining spaces of (1-3) with compatible homogeneous metrics. In this way, it is possible to construct many classes of homogeneous fibrations to which our results apply.

Twisted products. Let us now describe explicit triples $H \subsetneq K \subsetneq G$ of compact connected Lie groups with either $H$ normal in $K$ or $K$ normal in $G$. Starting with the latter, if a compact connected Lie group $G$ has a proper connected normal subgroup $K$, then there exists another connected normal subgroup ${ }^{6} L$ of $G$ such that $G=(K \times L) / \Gamma$, where $\Gamma \subset K \times L$ is finite. For any subgroup $H$ of $K$, one then gets the homogeneous fibration

$$
K / H \rightarrow((K \times L) / \Gamma) / H \rightarrow G / K .
$$

This provides an algorithm to build examples, whose input are the groups $H, K$, $L$ and $\Gamma$. Setting $G=(K \times L) / \Gamma$ we then have that the factor $K$ is a normal subgroup.

Example 5.1. Consider $G=\mathrm{SO}(4)$, which is double-covered by $S^{3} \times S^{3}$. In this case, $K=L=S^{3}$ and $\Gamma=\mathbb{Z}_{2}$ is the diagonal embedding into $K \times L$; that is, $\Gamma$ is the subgroup generated by $(-1,-1) \in S^{3} \times S^{3}$. We then have

$$
G=(K \times L) / \Gamma=\left(S^{3} \times S^{3}\right) / \mathbb{Z}_{2},
$$

and the quotient $G / K$ is isomorphic to $S^{3} / \mathbb{Z}_{2}=\mathrm{SO}(3)$. One can choose $H \subset K=S^{3}$ to be trivial, so that $K / H=S^{3}$; or to be a circle, for example the circle that gives the Hopf action on $S^{3}$, so that $K / H=S^{2}$. The corresponding homogeneous fibrations that (5-2) gives are

$$
S^{3} \rightarrow \mathrm{SO}(4) \rightarrow \mathrm{SO}(3) \text { and } S^{2} \rightarrow \mathrm{SO}(4) / S^{1} \rightarrow \mathrm{SO}(3) .
$$

In these cases the total space $G / H$ is a twisted product, while in the special case where $\Gamma$ is trivial we have the splitting $G=K \times L$, so that $G / K=L$ and $G / H=K / H \times L$ is an actual product manifold. That is, (5-2) becomes

$$
K / H \rightarrow K / H \times L \rightarrow L .
$$

\footnotetext{
${ }^{6}$ The subgroup $L$ is obtained as the connected subgroup of $G$ whose Lie algebra $\mathfrak{l}$ is the orthogonal complement (with respect to a bi-invariant metric) of the Lie algebra $\mathfrak{k}$ of $K$. Since $K$ is normal, $\mathfrak{k}$ is an ideal and then $\mathfrak{l}=\mathfrak{k}^{\perp}$ is also an ideal, because the adjoint representation is skew-symmetric with respect to the bi-invariant metric. This implies that $L$ is normal, and $K L$ generates the entire group $G$ by connectedness, since it does so locally near the identity. Finiteness of $\Gamma=K \cap L$ follows since the intersection $\mathfrak{k} \cap \mathfrak{l}$ is trivial.
} 
In contrast to the cases above, the deformed metrics $g_{t}$ in this situation are product metrics, obtained by rescaling the directions tangent to the first factor $K / H$ by $t^{2}$ and keeping the metric constant in the directions tangent to $L$. In this way, any product of a compact homogeneous space (with positive scalar curvature) and a compact connected Lie group satisfies the hypotheses of Theorem 4.1 (hence also of Theorem A). We note that this particular case is covered by the results of [Lima et al. 2012].

Sphere fibers. An important observation is that in the above product situation where $K / H=S^{n}$ is a sphere, our result allows for any homogeneous metric (not necessarily normal) on $K / H=S^{n}$ whose scalar curvature is positive, as opposed to only the round metric. Homogeneous metrics on spheres were classified by Ziller [1982]; they are obtained by rescaling the fibers of one of the Hopf fibrations:

$$
S^{1} \rightarrow S^{2 n+1} \rightarrow \mathbb{C} P^{n}, \quad S^{3} \rightarrow S^{4 n+3} \rightarrow \mathbb{H} P^{n}, \quad S^{7} \rightarrow S^{15} \rightarrow S^{8}\left(\frac{1}{2}\right) .
$$

In the first and last case, there is only one direction in which the fibers can be rescaled, while for the fibration with $S^{3}$ fiber, each of the 3 coordinate $S^{1}$ subgroups can be rescaled with a different factor. This gives rise to the following metrics:

- $\mathrm{g}_{s}$, a 1-parameter family of $\mathrm{U}(n+1)$-invariant metrics on $S^{2 n+1}$;

- $\mathrm{h}_{s_{1}, s_{2}, s_{3}}$, a 3-parameter family of $\operatorname{Sp}(n+1)$-invariant metrics on $S^{4 n+3}$;

- $\mathrm{k}_{s}$, a 1-parameter family of $\operatorname{Spin}(9)$-invariant metrics on $S^{15}$.

All these metrics have scal $>0$ for a certain range of parameters (see table below). Thus, if $K / H=S^{n}$ is isometric to one of those spheres, the submersion (5-3) satisfies the hypotheses of Theorem 4.1 (hence also of Theorem A).

\begin{tabular}{llll}
\hline metric & $K$ & $H$ & $\operatorname{dim} K / H$ \\
\hline \hline \multirow{3}{*}{ round } & $\mathrm{SO}(n+1)$ & $\mathrm{SO}(n)$ & $n(\geq 2)$ \\
& $\mathrm{Spin}(7)$ & $G_{2}$ & 7 \\
& $G_{2}$ & $\mathrm{SU}(3)$ & 6 \\
\hline \multirow{2}{*}{$\mathrm{g}_{s}$} & $\mathrm{SU}(n+1)$ & $\mathrm{SU}(n)$ & $2 n+1(\geq 2)$ \\
& $\mathrm{U}(n+1)$ & $\mathrm{U}(n)$ & $2 n+1(\geq 2)$ \\
\hline \multirow{2}{*}{$\mathrm{h}_{s_{1}, s_{2}, s_{3}}$} & $\mathrm{Sp}(n+1)$ & $\mathrm{Sp}(n)$ & $4 n+3$ \\
& $\mathrm{Sp}(n+1) \times \mathrm{Sp}(1)$ & $\mathrm{Sp}(n) \times \mathrm{Sp}(1)$ & $4 n+3$ \\
\hline $\mathrm{k}_{s}$ & $\mathrm{Sp}(n+1) \times \mathrm{U}(1)$ & $\mathrm{Sp}(n) \times \mathrm{U}(1)$ & $4 n+3$ \\
\hline
\end{tabular}

It is proved in [Bettiol and Piccione 2013, Proposition 4.2] that the range of 
parameters for which these $K$-invariant metrics have scal $>0$ is $0<s<s_{\max }$, where

$$
s_{\max }= \begin{cases}\sqrt{2 n+2} & \text { in the case of } \mathrm{g}_{s}, \\ \sqrt{\frac{2 n+4}{3}+\frac{\sqrt{18 n+16\left(n^{2}+2 n\right)^{2}}}{6 n}} & \text { in the case of } \mathrm{h}_{s, s, s}, \\ \sqrt{2+\frac{1}{2} \sqrt{19}} & \text { in the case of } \mathrm{k}_{s} .\end{cases}
$$

When $s=1$, these metrics are isometric to the round metric (which is the only normal homogeneous metric in each family).

Remark 5.2. The above construction can be interpreted as having a chain

$$
H \subsetneq H^{\prime} \subsetneq K \subsetneq G
$$

of Lie groups, and first performing a Cheeger deformation with respect to the $H^{\prime}$-action in the total space of $H^{\prime} / H \rightarrow K / H \rightarrow K / H^{\prime}$ to obtain positive scalar curvature on $K / H$, and then using this metric on the fiber of $K / H \rightarrow G / H \rightarrow G / K$. The Cheeger deformation with respect to the $K$-action on $G / H$ gives the desired 1-parameter family $g_{t}$ that satisfies the hypotheses of our results. More generally, one could perform multiple "preliminary" Cheeger deformations with a longer chain of groups in order to gain scal $>0$ on the fibers of the "last" homogeneous fibration.

Example 5.3. Another interesting class of examples with $K$ normal in $G$ is when $H$ is trivial, so that the resulting homogeneous fibration (1-3) is a short exact sequence of Lie groups $K \rightarrow G \rightarrow G / K$. This is precisely the case of $S^{3} \rightarrow \mathrm{SO}(4) \rightarrow \mathrm{SO}(3)$ in Example 5.1. Here, the deformed metrics $g_{t}$ are obtained by shrinking the original metric in the direction of the cosets of $K$ in $G$.

Other examples. As explained above, instead of having $K$ normal in $G$, one can also consider the case where $H$ is normal in $K$. This poses far fewer restrictions on the homogeneous fibrations that can be obtained, since the group $K$ will split as a product (up to a finite quotient); however, no conditions are imposed on $G$. For instance, $G$ may have arbitrarily large dimension and rank. It follows from the above discussion on normal homogenous metrics that our results apply to the submersion (1-3) with $H$ normal in $K$ as soon as the quotient $K / H$ is not abelian. More precisely, if $K / H$ is not a torus, then any normal homogeneous metric will have positive scalar curvature. Any choice of $G$ will then yield a triple $H \subsetneq K \subsetneq G$ whose corresponding homogeneous fibration can be endowed with compatible metrics for which Theorem 4.1 (hence also Theorem A) applies.

Example 5.4. To illustrate the above comments, let us build on the case described in Example 5.1. Instead of $G$, set $K=\operatorname{SO}(4)=\left(S^{3} \times S^{3}\right) / \mathbb{Z}_{2}$ and $H$ as one of the $S^{3}$ 
factors, so that $K / H=\mathrm{SO}(3)$. Then $G$ can be chosen arbitrarily among compact connected Lie groups that have a subgroup isomorphic to $\mathrm{SO}(4)$. As concrete examples, we may set $G=\mathrm{SO}(5)$ so that $G / K=S^{4}$ is a sphere; or $G=\mathrm{SO}(6)$ so that $G / K=T_{1} S^{4}$ is the unit tangent bundle of $S^{4}$. The corresponding homogeneous fibrations are

$$
\mathrm{SO}(3) \rightarrow \mathrm{SO}(5) / S^{3} \rightarrow S^{4} \quad \text { and } \quad \mathrm{SO}(3) \rightarrow \mathrm{SO}(6) / S^{3} \rightarrow T_{1} S^{4} .
$$

Remark 5.5. Any of the above examples can be trivially used to obtain new ones with nonsimply connected total space. Consider $F \subset K$ a finite subgroup and its action on $K / H$ and $G / H$, so that the inclusion map $K / H \rightarrow G / H$ is equivariant. One can form a new fibration replacing $K / H$ and $G / H$ by their (nonsimply connected) quotients by the $F$-action. Since $F \subset K$, the base of the fibration remains $G / K$. If the original metrics satisfied the conditions of Theorem 4.1, then the induced metrics in the new fibration also do.

\section{Bifurcation on nonhomogeneous fibrations}

A natural question is how the presence of many symmetries affects the bifurcation result obtained above. Homogeneity played a pivotal role in employing the equivariant bifurcation criterion (Proposition 2.3). When this assumption is dropped, the only tool at hand is the Morse index criterion (Proposition 2.2), so extra hypotheses are needed to guarantee a change in the Morse index at the degeneracy values. One such possibility is to impose certain curvature conditions that allow us to bound (from below) the growth of the eigenvalues of a nonhomogeneous collapsing Riemannian submersion.

Theorem 6.1. Let $F \rightarrow M \rightarrow B$ be a Riemannian submersion with totally geodesic fibers. Let $l=\operatorname{dim} F \geq 2$ and $m=\operatorname{dim} M$. Assume the metrics $g_{t}$ obtained by shrinking the fibers have constant scalar curvature, and that for some $\tau>0$ and $k_{1}, k_{2}>0$,

$$
\left\{\begin{array} { l } 
{ \operatorname { R i c } _ { F } \geq ( l - 1 ) k _ { 1 } } \\
{ \operatorname { s c a l } _ { F } < l ( m - 1 ) k _ { 1 } }
\end{array} \quad \text { and } \quad \left\{\begin{array}{l}
\operatorname{Ric}_{\left(M, g_{\tau}\right)} \geq(m-1) k_{2} \\
\operatorname{scal}_{B} \leq m(m-1) k_{2} .
\end{array}\right.\right.
$$

There exists a sequence $\left\{t_{q}\right\}$ in $] 0, \tau[$, that converges to 0 , of bifurcation values for the family $g_{t}$.

Proof. Since $\pi: M \rightarrow F$ is a Riemannian submersion with totally geodesic fibers,

$$
\operatorname{scal}\left(M, g_{t}\right)=\frac{1}{t^{2}} \operatorname{scal}_{F}+\operatorname{scal}_{B} \circ \pi-t^{2}\|A\|^{2},
$$

where $\|A\|$ is the Hilbert-Schmidt norm of the Gray-O'Neill tensor $A$. From $\operatorname{scal}_{F}>0$, we have $\lim _{t \rightarrow 0_{+}} \operatorname{scal}\left(M, g_{t}\right)=+\infty$. As before, the set of degeneracy values is discrete, and infinitely many of them occur due to $\operatorname{Spec}\left(\Delta_{B}\right) \subset \operatorname{Spec}\left(\Delta_{t}\right)$; 
see (3-5). Denote by $t_{q}$ the sequence of degeneracy values, accumulating at 0 , such that $\operatorname{scal}\left(M, g_{t_{q}}\right) /(m-1) \in \operatorname{Spec}\left(\Delta_{B}\right)$. We claim that for $q$ sufficiently large (that is, $t_{q}$ sufficiently small), $t_{q}$ is a bifurcation value.

From Proposition 2.2, we must verify that, for $t_{q}$ sufficiently small, there is a change of the Morse index $N\left(g_{t}\right)$ at $t_{q}$. It suffices to prove that every nonconstant eigenvalue $\lambda^{k, j}(t)$ of $\Delta_{t}$ is strictly larger than $\operatorname{scal}\left(M, g_{t}\right) /(m-1)$ for $t$ sufficiently small, so that no compensation of eigenvalues can occur (compare the proof of Theorem 4.1). Up to a simple rescaling, assume $\tau=1$. Since the eigenvalues $\mu_{k}$ of $\Delta_{M}$ and $\phi_{j}$ of $\Delta_{v}$ are ordered to be monotonically increasing, it suffices to prove (6-2) $\operatorname{scal}\left(M, g_{t}\right) /(m-1)<\lambda^{1,1}(t)=\mu_{1}+\left(\frac{1}{t^{2}}-1\right) \phi_{1}$ for $t$ sufficiently small;

see Proposition 3.2. From the Lichnerowicz estimates, since $\operatorname{Ric}_{F} \geq(l-1) k_{1}$ and $\operatorname{Ric}_{M} \geq(m-1) k_{2}$, we have

$$
\phi_{1} \geq l k_{1} \quad \text { and } \quad \mu_{1} \geq m k_{2}
$$

see [Chavel 1984, Chapter 3, Theorem 9]. When we combine the latter with $\operatorname{scal}_{B} \leq m(m-1) k_{2}$, we get

$$
\operatorname{scal}_{B} \circ \pi-t^{2}\|A\|^{2} \leq \operatorname{scal}_{B} \circ \pi \leq(m-1) \mu_{1} .
$$

Also, from (6-3) and $\operatorname{scal}_{F}<l(m-1) k_{1}$, it follows that $\operatorname{scal}_{F}<(m-1) \phi_{1}$. Thus, for $t$ sufficiently small, we have $\operatorname{scal}_{F}<\left(1-t^{2}\right)(m-1) \phi_{1}$, hence

$$
\frac{1}{t^{2}} \operatorname{scal}_{F}<(m-1)\left(\frac{1}{t^{2}}-1\right) \phi_{1} .
$$

Adding (6-4) and (6-5) and using (6-1), we obtain (6-2), concluding the proof.

The above result can be applied, for example, to low-dimensional Hopf fibrations, reobtaining the conclusion of Theorem 4.1. Nevertheless, for larger dimensions, the curvature pinching conditions are not satisfied, although the result remains true.

Remark 6.2. The curvature pinching conditions above are solely needed to avoid compensation of eigenvalues, in a rather forceful way. Given a Riemannian submersion $F \rightarrow M \rightarrow B$ with totally geodesic fibers of positive scalar curvature, suppose the metrics $g_{t}$ obtained by shrinking the fibers have constant scalar curvature. If under certain conditions there is an inclusion of (a nontrivial subgroup of) the isometry group of $F$ in the isometry group of $\left.\left(M, g_{t}\right), t \in\right] 0, \tau[$, then one can employ the equivariant techniques to deal with possible compensation of eigenvalues and still obtain infinitely many bifurcation values in this nonhomogeneous context.

\section{Multiplicity of solutions to the Yamabe problem}

We now explain how to obtain the multiplicity result claimed in the Introduction. 
Proposition 7.1. Let $g_{t}$, with $\left.t \in\right] 0, \tau$ [, be a family of metrics on $M$ with $N\left(g_{t}\right)>0$ and suppose there exists a sequence $\left\{t_{q}\right\}$ in $] 0, \tau[$, that converges to 0 , of bifurcation values for $g_{t}$. Then, there is an infinite subset $\left.\mathcal{T} \subset\right] 0, \tau[$ accumulating at 0, such that for each $t \in \mathcal{T}$, there are at least 3 solutions to the Yamabe problem in the conformal class $\left[g_{t}\right]$.

Proof. For all $t$, denote by $\hat{g}_{t}$ the unit volume metric homothetic to $g_{t}$. Since $t_{q}$ is a bifurcation value, there are values of $t$ arbitrarily close to $t_{q}$ for which the conformal class $\left[g_{t}\right]$ contains a unit volume constant scalar curvature metric $g$ distinct from $\hat{g}_{t}$. Since $N\left(g_{t}\right)>0$, by continuity of the Morse index, also $N(g)>0$. In particular, neither $\hat{g}_{t}$ nor $g$ are minima of the Hilbert-Einstein functional in $\left[g_{t}\right]$. Therefore, $\left[g_{t}\right]$ contains at least 3 distinct unit volume constant scalar curvature metrics, that is, 3 solutions to the Yamabe problem. The set $\mathscr{T}$ of such $t$ 's clearly accumulates at 0 , since $t_{q}$ converges to 0 .

Theorems A and B now follow easily from Theorems 4.1 and 6.1, respectively. Indeed, in order to apply Proposition 7.1, it is only necessary to verify that $N\left(g_{t}\right)>0$. In the first case, if $g_{t}$ comes from a homogeneous fibration, there must be a value $\tau$ of $t$, when $\operatorname{scal}(t) /(m-1)$ crosses the first eigenvalue from the base, before any compensation is even possible. At this value $t=\tau$, the Morse index changes from 0 to a positive integer. Then, for $t \in] 0, \tau$ [, we have $N\left(g_{t}\right) \geq N\left(g_{\tau-\varepsilon}\right)>0$. In the second case, $N\left(g_{t}\right)$ gets arbitrarily large as $t \rightarrow 0$, so this condition is also satisfied.

\section{References}

[Ambrosetti and Malchiodi 1999] A. Ambrosetti and A. Malchiodi, "A multiplicity result for the Yamabe problem on $S^{n}$ ”, J. Funct. Anal. 168:2 (1999), 529-561. MR 2000k:53032 Zbl 0949.53028

[Anderson 2005] M. T. Anderson, "On uniqueness and differentiability in the space of Yamabe metrics”, Commun. Contemp. Math. 7:3 (2005), 299-310. MR 2006d:53031 Zbl 1082.58013

[Aubin 1976] T. Aubin, "Équations différentielles non linéaires et problème de Yamabe concernant la courbure scalaire”, J. Math. Pures Appl. (9) 55:3 (1976), 269-296. MR 55 \#4288 Zbl 0336.53033

[Bérard-Bergery 1978] L. Bérard-Bergery, "Sur la courbure des métriques riemanniennes invariantes des groupes de Lie et des espaces homogènes", Ann. Sci. École Norm. Sup. (4) 11:4 (1978), 543-576. MR 80k:53078 Zbl 0426.53038

[Bérard-Bergery and Bourguignon 1982] L. Bérard-Bergery and J.-P. Bourguignon, "Laplacians and Riemannian submersions with totally geodesic fibres", Illinois J. Math. 26:2 (1982), 181-200. MR 84m:58153 Zbl 0483.58021

[Berti and Malchiodi 2001] M. Berti and A. Malchiodi, "Non-compactness and multiplicity results for the Yamabe problem on $S^{n}$ ”, J. Funct. Anal. 180:1 (2001), 210-241. MR 2002b:53049 Zbl 0979.53038

[Bessa et al. 2012] G. P. Bessa, J. F. Montenegro, and P. Piccione, "Riemannian submersions with discrete spectrum”, J. Geom. Anal. 22:2 (2012), 603-620. MR 2891739 Zbl 1257.58018

[Besse 1987] A. L. Besse, Einstein manifolds, Results in Mathematics and Related Areas (3) 10, Springer, Berlin, 1987. MR 88f:53087 Zbl 0613.53001 
[Bettiol and Piccione 2013] R. G. Bettiol and P. Piccione, "Bifurcation and local rigidity of homogeneous solutions to the Yamabe problem on spheres", Calc. Var. Partial Differential Equations 47:3-4 (2013), 789-807. MR 3070564

[Chavel 1984] I. Chavel, Eigenvalues in Riemannian geometry, Pure and Applied Mathematics 115, Academic Press, Orlando, FL, 1984. MR 86g:58140 Zbl 0551.53001

[Fischer and Marsden 1975] A. E. Fischer and J. E. Marsden, "Deformations of the scalar curvature", Duke Math. J. 42:3 (1975), 519-547. MR 52 \#1804 Zbl 0336.53032

[Kielhöfer 2004] H. Kielhöfer, Bifurcation theory: An introduction with applications to PDEs, Applied Mathematical Sciences 156, Springer, New York, 2004. MR 2004i:47133 Zbl 1032.35001 [Lima et al. 2012] L. L. de Lima, P. Piccione, and M. Zedda, "On bifurcation of solutions of the Yamabe problem in product manifolds", Ann. Inst. H. Poincaré Anal. Non Linéaire 29:2 (2012), 261-277. MR 2901197 Zbl 1239.58005

[Pollack 1993] D. Pollack, "Nonuniqueness and high energy solutions for a conformally invariant scalar equation”, Comm. Anal. Geom. 1:3-4 (1993), 347-414. MR 94m:58051 Zbl 0848.58011

[Schoen 1984] R. Schoen, "Conformal deformation of a Riemannian metric to constant scalar curvature”, J. Differential Geom. 20:2 (1984), 479-495. MR 86i:58137 Zbl 0576.53028

[Schoen 1989] R. M. Schoen, "Variational theory for the total scalar curvature functional for Riemannian metrics and related topics", pp. 120-154 in Topics in calculus of variations (Montecatini Terme, 1987), edited by M. Giaquinta, Lecture Notes in Math. 1365, Springer, Berlin, 1989. MR 90g:58023 Zbl 0702.49038

[Schoen 1991] R. M. Schoen, "On the number of constant scalar curvature metrics in a conformal class", pp. 311-320 in Differential geometry, edited by B. Lawson and K. Tenenblat, Pitman Monogr. Surveys Pure Appl. Math. 52, Longman Sci. Tech., Harlow, 1991. MR 94e:53035 Zbl 0733.53021

[Smoller and Wasserman 1990] J. Smoller and A. G. Wasserman, "Bifurcation and symmetrybreaking”, Invent. Math. 100:1 (1990), 63-95. MR 91f:58019 Zbl 0721.58011

[Trudinger 1968] N. S. Trudinger, "Remarks concerning the conformal deformation of Riemannian structures on compact manifolds", Ann. Scuola Norm. Sup. Pisa (3) 22 (1968), 265-274. MR 39 \#2093 Zbl 0159.23801

[Yamabe 1960] H. Yamabe, "On a deformation of Riemannian structures on compact manifolds", Osaka Math. J. 12 (1960), 21-37. MR 23 \#A2847 Zbl 0096.37201

[Ziller 1982] W. Ziller, "Homogeneous Einstein metrics on spheres and projective spaces", Math. Ann. 259:3 (1982), 351-358. MR 84h:53062 Zbl 0469.53043

Received August 6, 2012.

RENATO G. BETTIOL

DEPARTMENT OF MATHEMATICS

UNIVERSITY OF NOTRE DAME

Notre DAME, IN 46556-4618

UNITED STATES

rbettiol@nd.edu

\section{PaOlo Piccione}

Departamento de Matemática

UNIVERSIDADE DE SÃo PAULO

SÃo PAUlO, SP, 05508-090

BRAZIL

piccione@ime.usp.br 


\title{
PACIFIC JOURNAL OF MATHEMATICS
}

\author{
msp.org/pjm
}

Founded in 1951 by E. F. Beckenbach (1906-1982) and F. Wolf (1904-1989)

\section{EDITORS}

V. S. Varadarajan (Managing Editor)

Department of Mathematics

University of California

Los Angeles, CA 90095-1555

pacific@math.ucla.edu

Paul Balmer

Department of Mathematics

University of California

Los Angeles, CA 90095-1555

balmer@math.ucla.edu

Daryl Cooper

Department of Mathematics

University of California

Santa Barbara, CA 93106-3080 cooper@math.ucsb.edu

Jiang-Hua $\mathrm{Lu}$

Department of Mathematics

Pokfulam Rd., Hong Kong jhlu@maths.hku.hk
The University of Hong Kong

Don Blasius

Department of Mathematics University of California

Los Angeles, CA 90095-1555

blasius@math.ucla.edu

Robert Finn

Department of Mathematics Stanford University

Stanford, CA 94305-2125

finn@math.stanford.edu

Sorin Popa

Department of Mathematics

University of California

Los Angeles, CA 90095-1555 popa@math.ucla.edu

Paul Yang

Department of Mathematics Princeton University

Princeton NJ 08544-1000

yang@math.princeton.edu

\section{PRODUCTION}

Silvio Levy, Scientific Editor, production@msp.org

\section{SUPPORTING INSTITUTIONS}

ACADEMIA SINICA, TAIPEI

CALIFORNIA INST. OF TECHNOLOGY

INST. DE MATEMÁTICA PURA E APLICADA

KEIO UNIVERSITY

MATH. SCIENCES RESEARCH INSTITUTE

NEW MEXICO STATE UNIV.

OREGON STATE UNIV.

\author{
STANFORD UNIVERSITY \\ UNIV. OF BRITISH COLUMBIA \\ UNIV. OF CALIFORNIA, BERKELEY \\ UNIV. OF CALIFORNIA, DAVIS \\ UNIV. OF CALIFORNIA, LOS ANGELES \\ UNIV. OF CALIFORNIA, RIVERSIDE \\ UNIV. OF CALIFORNIA, SAN DIEGO \\ UNIV. OF CALIF., SANTA BARBARA
}

\author{
Vyjayanthi Chari \\ Department of Mathematics \\ University of California \\ Riverside, CA 92521-0135 \\ chari@math.ucr.edu \\ Kefeng Liu \\ Department of Mathematics \\ University of California \\ Los Angeles, CA 90095-1555 \\ liu@math.ucla.edu \\ Jie Qing \\ Department of Mathematics \\ University of California \\ Santa Cruz, CA 95064 \\ qing@cats.ucsc.edu
}

These supporting institutions contribute to the cost of publication of this Journal, but they are not owners or publishers and have no responsibility for its contents or policies.

See inside back cover or msp.org/pjm for submission instructions.

The subscription price for 2013 is US \$400/year for the electronic version, and \$485/year for print and electronic.

Subscriptions, requests for back issues and changes of subscribers address should be sent to Pacific Journal of Mathematics, P.O. Box 4163, Berkeley, CA 94704-0163, U.S.A. The Pacific Journal of Mathematics is indexed by Mathematical Reviews, Zentralblatt MATH, PASCAL CNRS Index, Referativnyi Zhurnal, Current Mathematical Publications and the Science Citation Index.

The Pacific Journal of Mathematics (ISSN 0030-8730) at the University of California, c/o Department of Mathematics, 798 Evans Hall \#3840, Berkeley, CA 94720-3840, is published twelve times a year. Periodical rate postage paid at Berkeley, CA 94704, and additional mailing offices. POSTMASTER: send address changes to Pacific Journal of Mathematics, P.O. Box 4163, Berkeley, CA 94704-0163.

PJM peer review and production are managed by EditFLOW ${ }^{\circledR}$ from Mathematical Sciences Publishers.

\section{PUBLISHED BY}

mathematical sciences publishers

nonprofit scientific publishing

http://msp.org/

(C) 2013 Mathematical Sciences Publishers 


\section{PACIFIC JOURNAL OF MATHEMATICS}

Volume $266 \quad$ No. $1 \quad$ November 2013

Multiplicity of solutions to the Yamabe problem on collapsing $\quad 1$ Riemannian submersions

Renato G. Bettiol and Paolo Piccione

Rank gradient of small covers

DARLAN GIRÃO

Nonrationality of nodal quartic threefolds

KYUSIK HONG

Supertropical linear algebra

ZUR IZHAKIAN, MANFRED KNEBUSCH and LOUIS ROWEN

Isometry groups among topological groups

PIOTR NIEMIEC

Singularities and Liouville theorems for some special conformal

Hessian equations

QIANZHONG OU

Attaching handles to Delaunay nodoids

FRANK PACARD and HAROLD ROSENBERG

Some new canonical forms for polynomials

BRUCE REZNICK

Applications of the deformation formula of holomorphic one-forms 